\title{
Effect of Acupressure on Primary Dysmenorrhea: Review of Experimental Studies
}

\author{
Aslı Karakuş Selçuk*, Emre Yanikkerem \\ Department of Nursing, Faculty of Health Science, Manisa Celal Bayar University, Manisa, Turkey
}

Received June 24, 2020

Revised January 16, 2021

Accepted March 8, 2021

Correspondence to Aslı Karakuş Selçuk Department of Nursing, Faculty of Health Science, Manisa Celal Bayar University, Manisa, Turkey

E-mail akarakus_ksk@hotmail.com
Primary dysmenorrhea is defined as cramping pain in the lower abdomen with no pelvic diseases, and it has a high prevalence in many countries. Acupressure is a widely used complementary treatment method for primary dysmenorrhea. This review examined experimental studies to determine the effects of acupressure on primary dysmenorrhea using the databases PubMed, Google Scholar, and CINAHL with the keywords "Acupressure" and "Dysmenorrhea". There were 2227 records in the databases, and 330 articles were published between 1989 and March 2020. Experimental studies in the English language were reviewed according to the PRISMA guidelines. This review included 28 published studies that were assessed using the Jadad score for quality. The studies were categorized as studies of acupressure at the LR3 point $(n=4)$, at the SP6 point $(n=9)$, at auricular points $(n=5)$, at multiple points $(n=8)$, and with devices $(n=2)$. Moreover, studies of self-acupressure $(n=9)$ were identified. The studies demonstrated that acupressure could reduce menstrual symptoms, the severity and duration of menstrual pain, distress, and anxiety. Furthermore, it helped improve the quality of life and well-being of patients and provide psychological support and self-care. Acupressure is an inexpensive, easy-to-apply, and non-pharmacological treatment and is useful for reducing primary dysmenorrhea, and women can apply this method anywhere by themselves. However, high-quality randomized controlled trials with larger samples are necessary to establish the evidence for acupressure as an effective intervention.

Keywords: Acupressure, Primary dysmenorrhea, Acupoints, Self-acupressure

\section{INTRODUCTION}

Primary dysmenorrhea (PD) is defined as cramping pain in the lower abdomen, which occurs before or during menstruation without pelvic diseases [1-4]. Excessive prostaglandin release causes uterine contraction and leads to uterine hypoxia and ischemia, which results in extreme abdominal cramps and pain $[1,3,5]$.

The rates of $\mathrm{PD}$ are high in some countries, e.g., $85.7 \%$ in Saudi Arabia [6], 74.8\% in Spain [7], 80.0\% in China [8], $88.0 \%$ in Australia [9], and between $84.0 \%$ and $94.0 \%$ in Turkey [10-13]. Contraceptive pills and nonsteroidal antiinflammatory drugs are commonly used in the treatment of PD $[3,5,14,15]$. Acupressure is a traditional Chinese medicine technique involving the use of fingers and palms for noninvasive nursing intervention [1,16-21]. The method is also easy to learn and teach, low-cost, and simple and does not have negative side effects, and every person can utilize this method anywhere by themselves [1,16-19]. Acupressure is one of the complementary treatment methods for PD, which is widely used $[14,20,22]$. It can activate the flow of body energy and blood, stimulate endorphin production, and lead to pain relief [23-25]. In addition, acupressure can reduce the social and economic consequences of PD $[17,26]$. Therefore, this review examined experimental studies to determine the effects of acupressure on PD.

\section{MATERIALS AND METHODS}

\section{Searching strategy and selection of studies}

Articles related to the effect of acupressure on PD published between 1989 and March 2020 were reviewed using the PubMed, Google Scholar, and Cumulative Index to Nursing and Allied Health Literature (CINAHL) databases with the keywords "Acupressure" and "Dysmenorrhea". The Preferred Reporting Items for Systematic Review and MetaAnalysis (PRISMA) declaration guided the study process [27]. Eligibility assessment was performed independently 
in a blinded standardized manner by the researchers. The authors independently reviewed all abstracts and titles. The inclusion criteria in this study were as follows: experimental human studies, studies in the English language, and full-text studies. Review articles, studies not in the English language, articles with descriptive, cross-sectional, and qualitative methods, letter to the editor, congress presentations, books, study protocols, and case reports were excluded from the review. There were 2227 records in the databases, and 330 articles were examined. Overall, 28 published studies met the inclusion criteria for this review. Details of the article selection process for the review are shown in Fig. 1.

\section{Quality assessment}

The quality of each study was assessed using the Jadad score, which is a tool used to assess the methodological quality of a clinical trial independently. Similar systematic reviews were assessed using this score $[28,29]$. The total

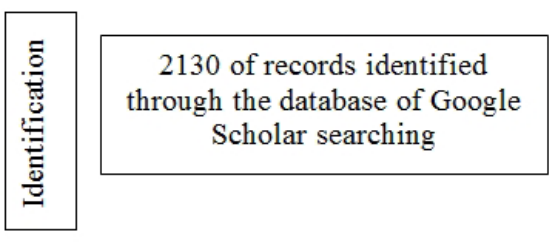

57 of records identified through the database of Pubmed searching
56 of records identified through the database of CINAHL searching

2227 of records after duplicates removed

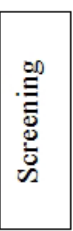

2227 of records screened 1875 of records excluded those reasons: Reviews: 1456

Not in English: 349

Descriptive, cross-sectional and qualitative methods: 54
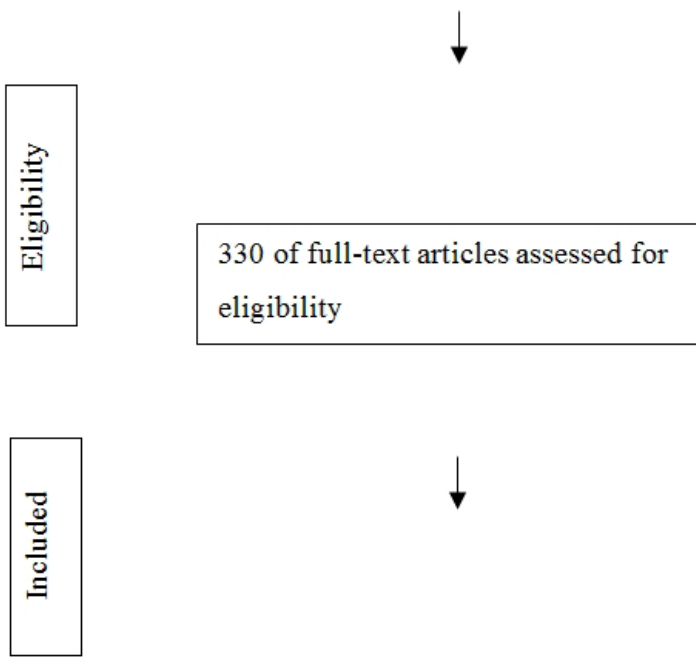

Letter to the editor: 5 , Congress presentation: 4

Book: 3, Study protocol: 3, Case report: 1

302 of full-text articles were excluded due to the effect of acupressure on other diseases such as weight reduction, abdominal obesity, hyperemesis gravidarum, etc., other complementary therapies on dysmenorrhea such as aromatherapy, yoga, and reflexology, etc., the effect of acupressure on quality of life and detecting the tenderness occurrence rate.

28 of studies included in the review

(Pubmed: 23, Google Scholar: 4, CINAHL: 1)

Fig. 1. Details of the article selection in process of the review. 


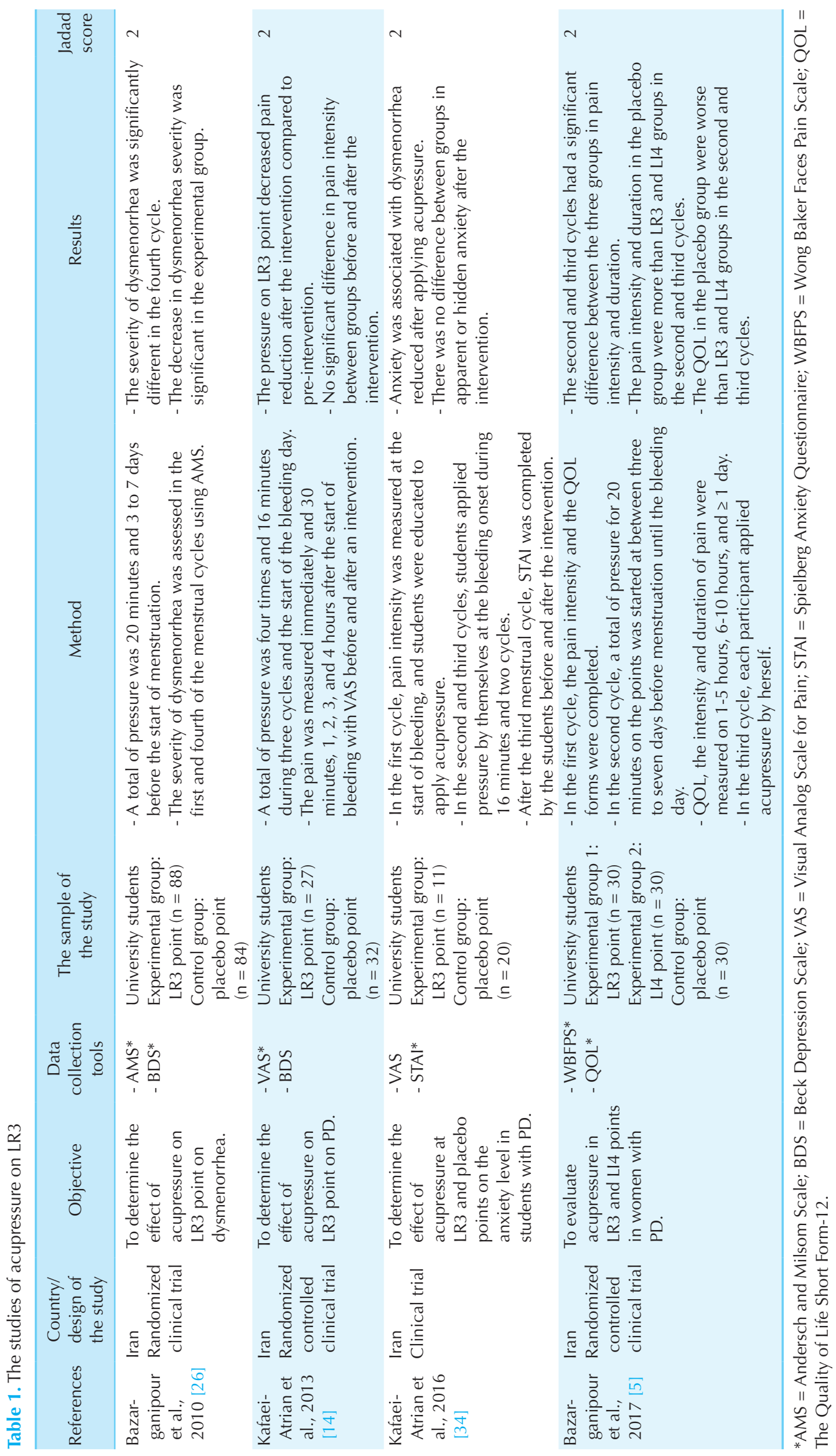




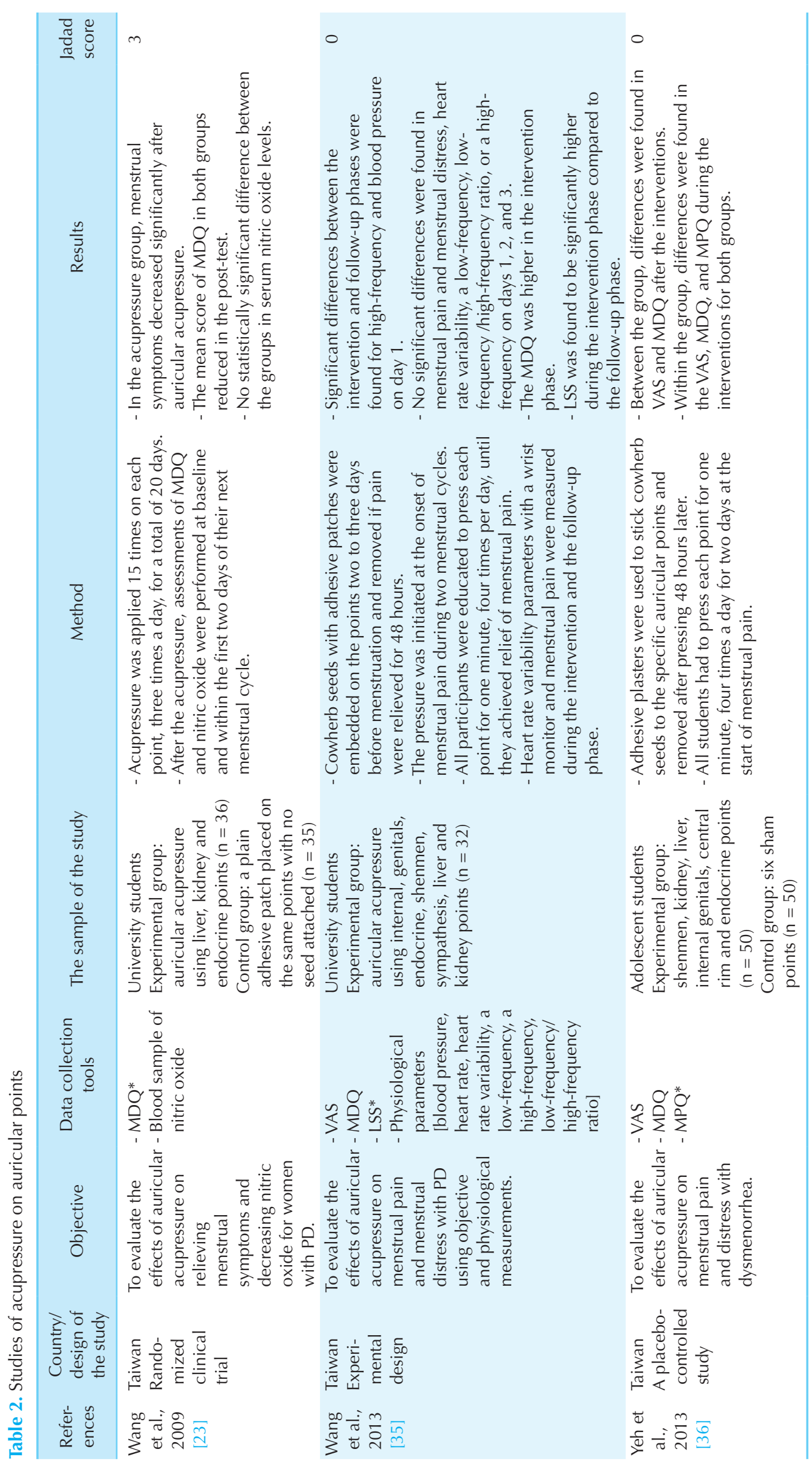




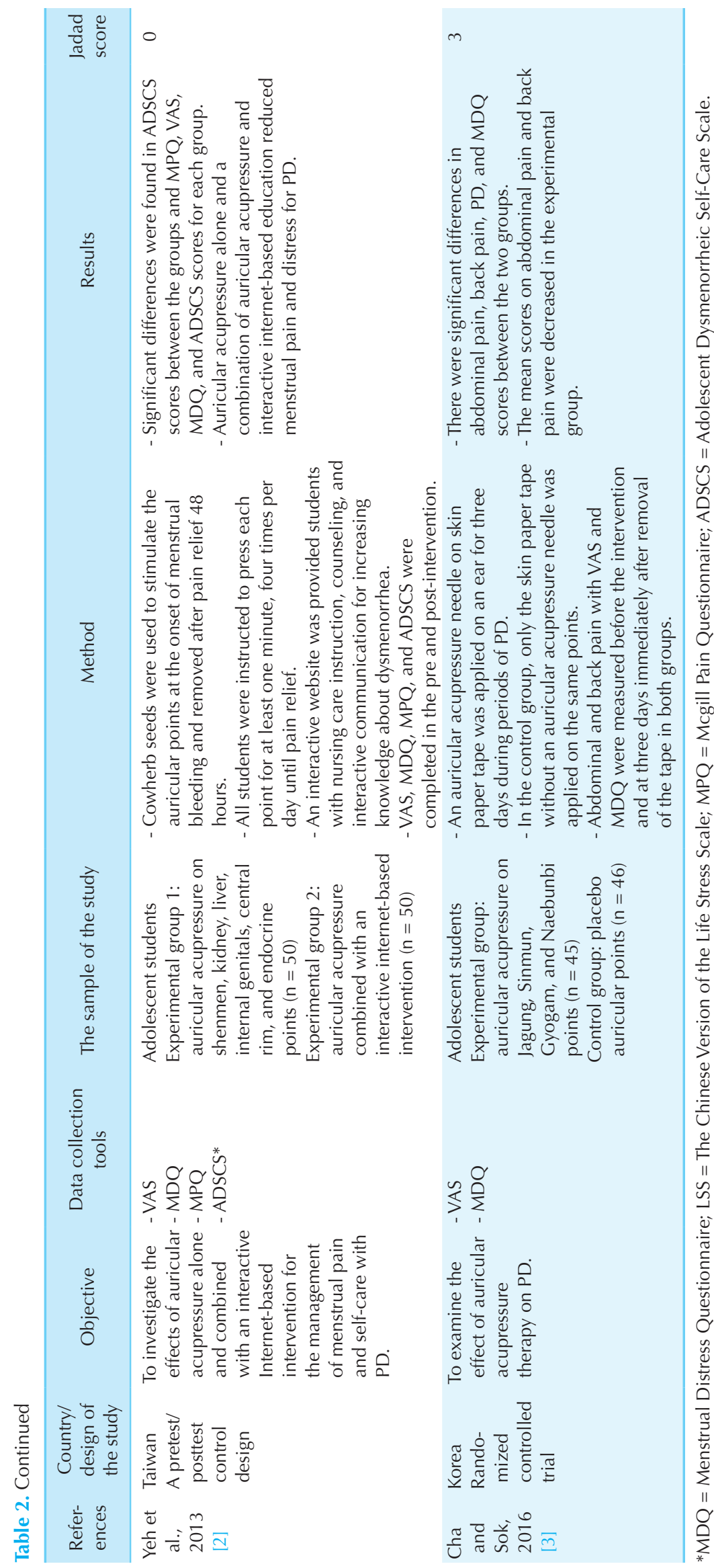




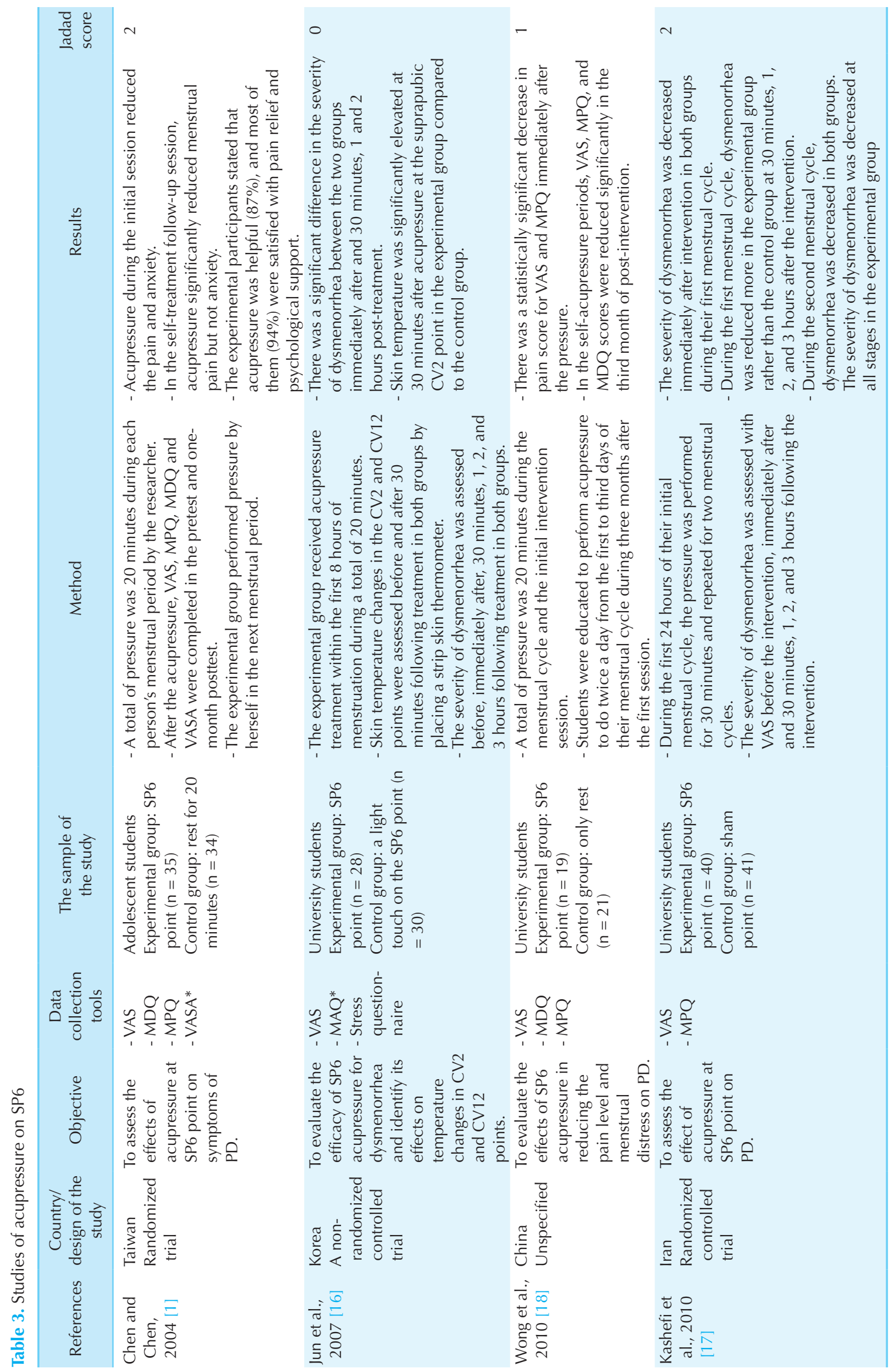









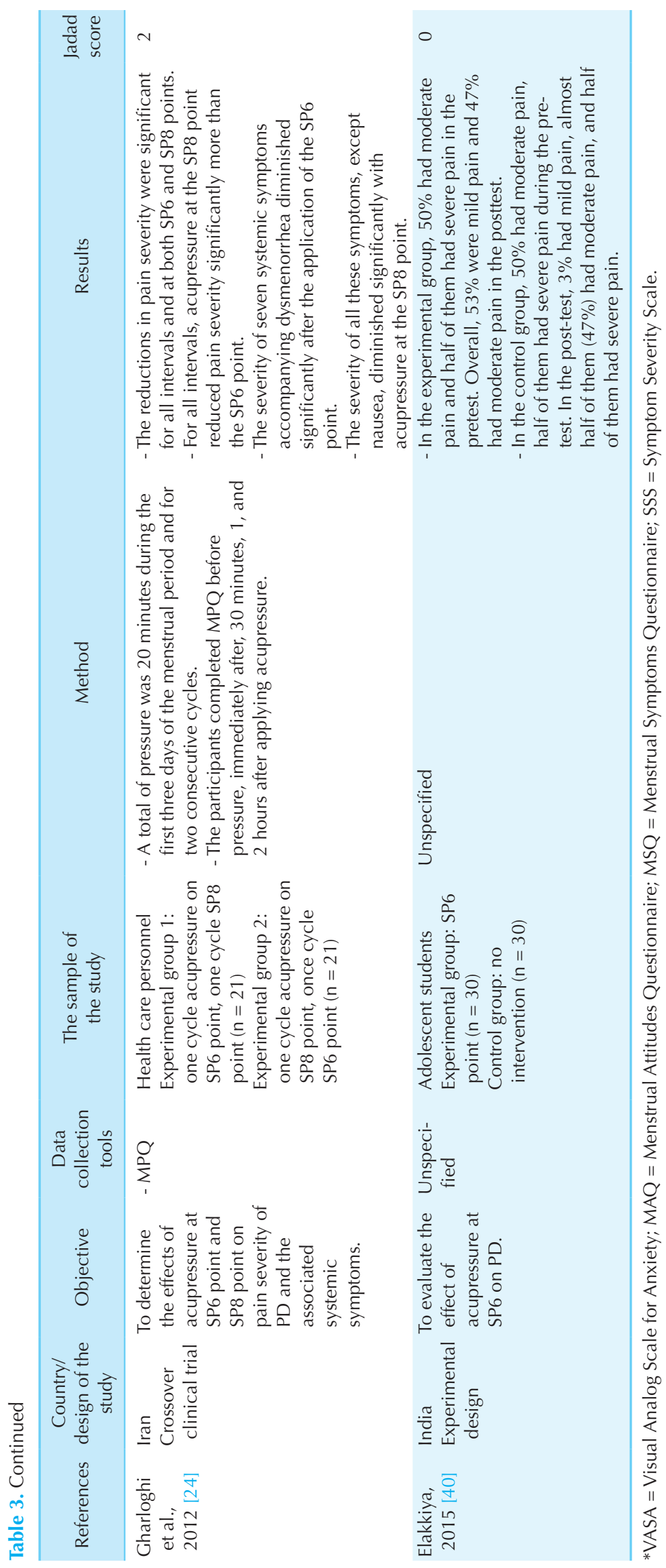









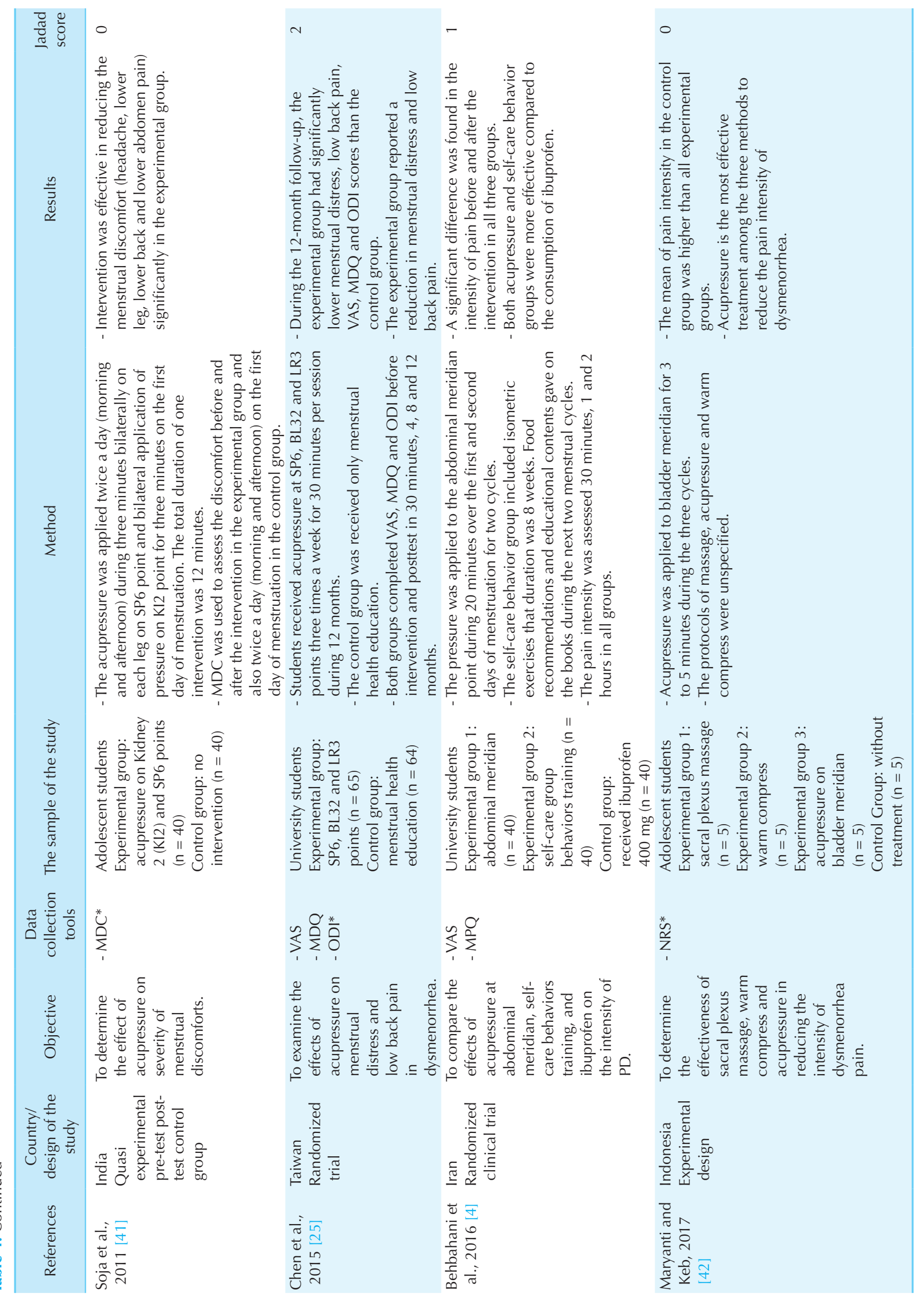




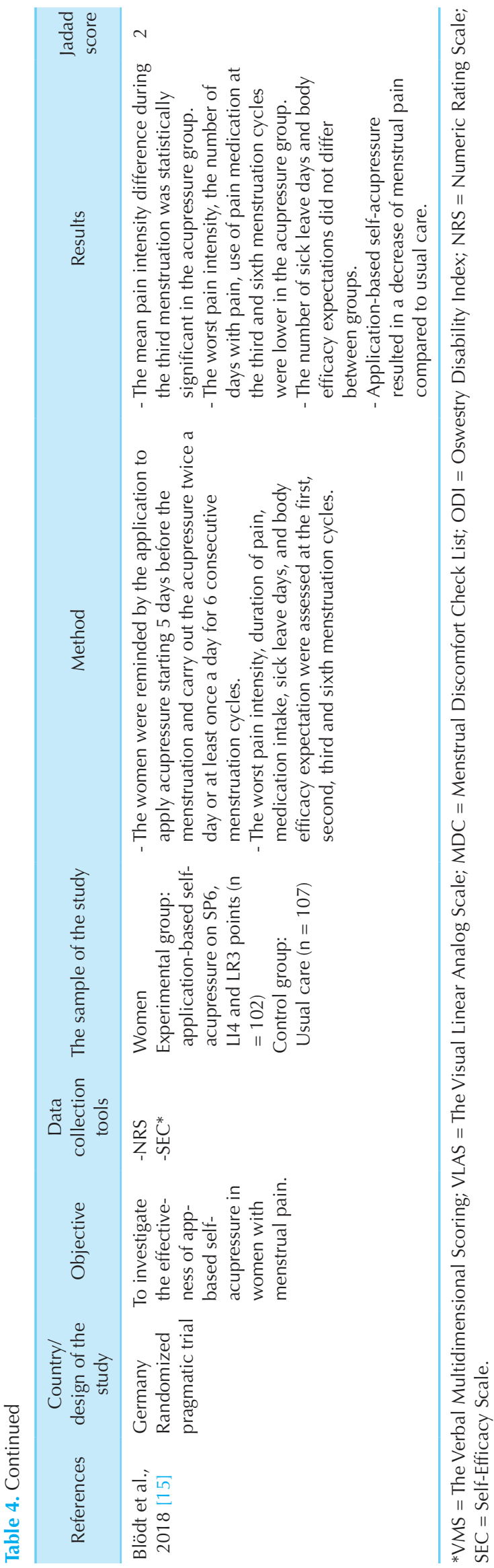

score ranged from 0 to 5 points. A study was considered high quality if it scored 2 or higher and low quality if it scored lower than $2[30,31]$. The methodological quality of all studies was also evaluated separately by two reviewers.

\section{Features of the studies}

The characteristics of the 28 studies are presented in Tables 1, 2, 3, 4, and 5. The studies were conducted in 8 different countries, mostly in Iran $[4,5,14,17,19,20,24,26,32$ 34] and Taiwan $[1,2,22,23,25,35,36]$. The other countries were Germany [15], China [18], Korea [3,16], United States of America (USA) [37,38], India [39-41], and Indonesia [42]. The sample size of the studies ranged from 20 to 216 women. Of the studies, 23 studies involved students, 4 studies involved women, and 1 study involved health care personnel.

The same inclusion criteria were used in all studies for acupressure as follows: duration of bleeding between 3 and 8 days, menstrual intervals of 21 and 35 days, lack of any disease and abdominal/pelvic surgery, absence of any severe psychological stress, no usage of oral or other contraceptives or drugs, absence of any lesion, varices, or inflammatory skin disease at the location of pressure application, and no genital disease.

\section{RESULTS}

The studies were categorized as studies of acupressure at the LR3 point ( $\mathrm{n}=4$ articles), at the SP6 point ( $\mathrm{n}=9$ articles), at auricular points ( $\mathrm{n}=5$ articles), at multiple points ( $\mathrm{n}=8$ articles), and with devices ( $\mathrm{n}=2$ articles). Moreover, studies of self-acupressure $(n=9)$ were identified. The World Health Organization standard nomenclature for acupuncture points was used [43].

\section{Studies of acupressure at the LR3 point}

Four studies were conducted with university students in Iran. The severity of dysmenorrhea in the first and last cycles was compared to that when acupressure was applied in the LR3 and placebo groups to evaluate the pain intensity $[5,14,26]$ and anxiety level [34]. Three studies found that acupressure at the LR3 point decreased the pain intensity, and pain reduction was determined by comparing postintervention with pre-intervention pain $[5,14,26]$. In two of the studies, students were taught acupressure techniques so that they can apply acupressure themselves, and they found that acupressure could relieve pain during menstruation [5] and help reduce anxiety [34].

\section{Studies of acupressure at auricular points}

Studies involving students in Taiwan were performed to investigate the effects of auricular acupressure on $\mathrm{PD}$ 


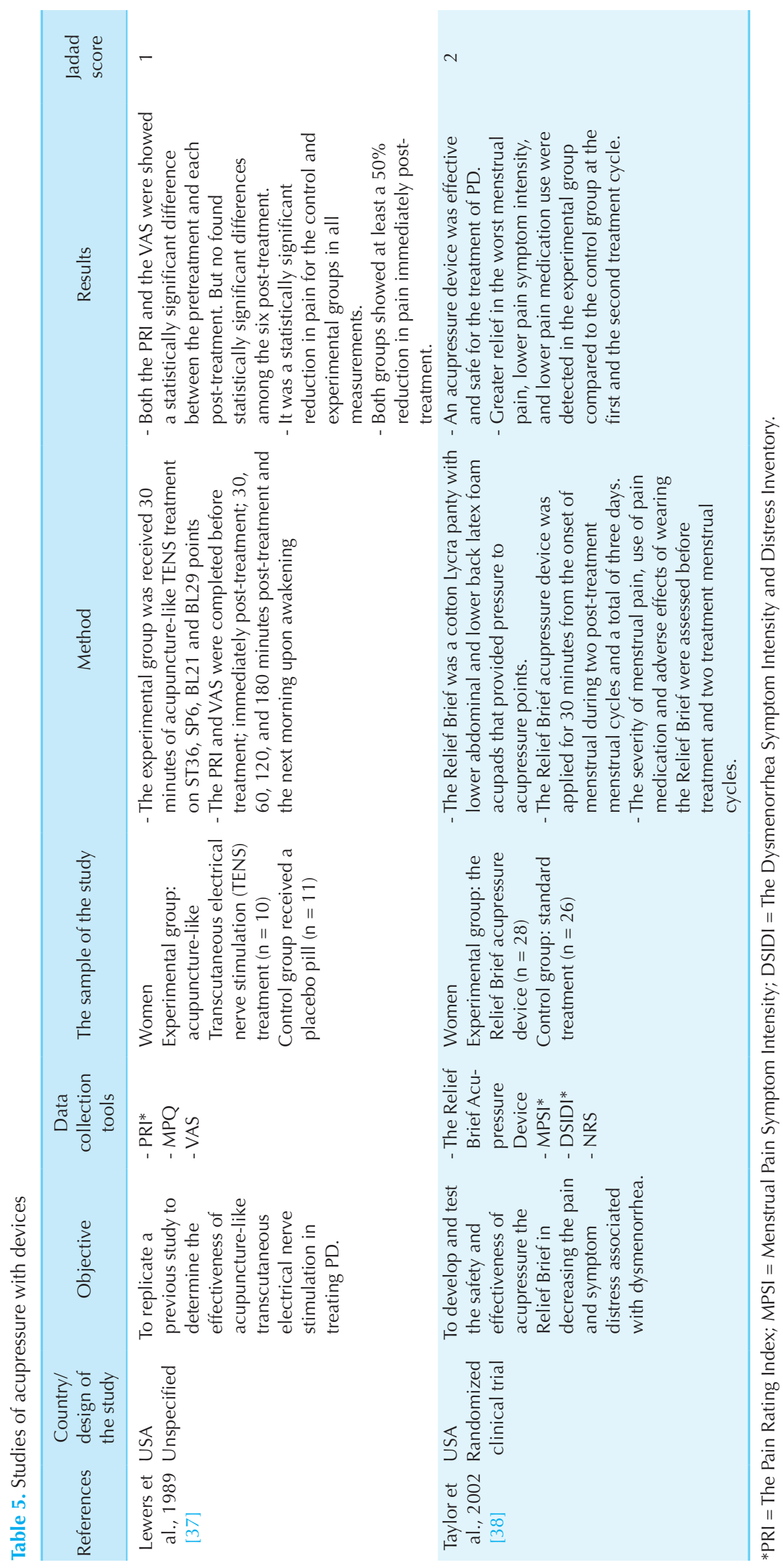


using cowherb seeds with adhesive patches, which were embedded at auricular points $(n=4)[2,23,35,36]$. In a study in Korea, needles on skin paper tape were applied to the ear $(\mathrm{n}=1)$ [3]. The Menstrual Distress Questionnaire was used to evaluate dysmenorrhea severity, and liver, kidney, and endocrine auricular points were commonly used to relieve dysmenorrhea in the studies.

Dysmenorrhea is known to be associated with stress, which increases the severity of pain with activation of sympathetic responses and inhibition of parasympathetic responses. Both stress and pain activate the sympathetic nervous system, which increases heart rate, muscle contraction, and blood pressure. In a study of auricular acupressure in Taiwan, there were significant differences between the intervention and follow-up phases for the high-frequency component in heart rate variability and blood pressure. Moreover, the study showed that auricular acupressure increased parasympathetic activity. It is beneficial to maintain autonomic function homeostasis to relieve menstrual pain and distress under stress conditions [35]. According to three studies, the effectiveness of acupressure at auricular points was superior to acupressure at placebo auricular points in decreasing the menstrual symptoms of women $[3,23,36]$. In a study in Taiwan, auricular acupressure with an interactive internetbased intervention was given to students to increase their knowledge about dysmenorrhea. The combination intervention was better than auricular acupuncture alone in improving self-care behaviors and reducing menstrual pain and distress in $\mathrm{PD}$ [2].

\section{Studies of acupressure at the SP6 point}

The effects of acupressure at the SP6 point on PD were investigated in five studies in Iran $[17,19,20,24,33]$ as well as studies in India [40], Taiwan [1], China [18], and Korea [16].

The studies found that acupressure alleviated menstrual pain during the initial and follow-up sessions in the experimental group compared with the control group with no intervention $[1,18,19,40]$. Two studies demonstrated that acupressure reduced menstrual pain in students who were educated on self-acupressure techniques $[1,18]$. In addition, three studies showed that the severity of dysmenorrhea was markedly decreased in the experimental group compared with the control group $[16,17,20]$.

In another study, the severity of dysmenorrhea and skin temperature changes at the CV2 and CV12 acupoints before treatment and $0.5,1,2$, and 3 hours following treatment were evaluated in both the experimental and control groups. The results showed that skin temperature was significantly elevated at the suprapubic CV2 point after acupressure at SP6 in the experimental group, and there was an increase in blood flow to the lower abdomen [16].
A study in Iran compared the average pain intensity between the acupressure, fish oil, and ibuprofen groups. The group taking ibuprofen was the most satisfied, followed by the acupressure and the fish oil groups, and all groups needed the same quantity of extra painkillers during the treatment duration [33].

A study compared acupressure at the SP6 and SP8 points before, immediately after, and $0.5,1$, and 2 hours after applying acupressure. It was found that the reductions in pain severity were significant for all measurements at the SP6 and SP8 points; however, pain intensity was more effectively reduced by acupressure at the SP8 point compared with the SP6 point. In addition, the severity of seven systemic symptoms (exhaustion, vomiting, paralysis, diarrhea, headache, neural, and faint) accompanying dysmenorrhea was reduced significantly after acupressure at the SP6 and SP8 points [24].

\section{Studies of acupressure at multiple points}

Seven of the studies applied acupressure at multiple points, including LI4, SP15, ST36, SP6, and LR3 in an Iranian study [32], SP6, LI4, and ST36 in a Taiwanese study [22], SP6, LR4, and ST41 in an Indian study [39], SP6 and KI2 in an Indian study [41], SP6, BL32, and LR3 in a Taiwanese study [25], the abdominal meridian in an Iranian study [4], the bladder meridian in an Indonesian study [42], and SP6, LI4, and LR3 in a German study [15].

A study in Iran assessed pain intensity in three groups: acupressure, self-care behavior (isometric exercise, food recommendations, and educational contents), and ibuprofen. The study found that there was a significant difference in the mean intensity of pain before and after the intervention in all three groups; however, both acupressure and self-care behavior were more effective compared with ibuprofen [4].

In Iran, a study comparing the pain in three groups (sham acupressure as a placebo, acupressure, and ibuprofen) reported that the three therapeutic techniques were highly effective in reducing pain. In addition, acupressure was significantly better than placebo, and acupressure and ibuprofen showed similar effects [32].

A study in Indonesia found that the most effective treatment to reduce pain intensity in dysmenorrhea was acupressure compared with warm compress or sacral plexus massage [42]. During a 12-month follow-up period in a study in Taiwan, menstrual distress and back pain were significantly lower in the acupressure group compared with the control group, which received only a menstrual health education [25].

During a 6-month follow-up period, a randomized trial was conducted in Taiwan with adolescent students divided into three experimental groups for self-acupressure (group 
1: ST36, group 2: LI4, and group 3: LI4 and SP6 matched points) and a control group with rest for 20 minutes without treatment. Pain, distress, and anxiety were reduced in group 3, and menstrual pain was alleviated in group 2 [22].

In a study involving women in India, changes in heart rate variability (high-frequency component, low-frequency component, low-frequency/high-frequency ratio) and pain intensity were assessed before and after acupressure in three groups. In group 1 and group 2, heart rate variability and VAS were measured when experiencing pain during menstruation. In group 2, after acupressure was applied at the SP6, LR4, and ST41 points during pain, all variables were measured again. In the control group, heart rate variability was assessed for women who did not experience dysmenorrhea. The pain intensity and high-frequency component were significantly decreased after acupressure, whereas the low-frequency component and low-frequency/high-frequency ratio were increased. The study demonstrated that acupressure was effective in regulating autonomic alterations [39]. In another study involving students in India, the effect of acupressure on the severity of menstrual discomfort was determined in two groups. Acupressure was applied in the morning and afternoon bilaterally on each leg at the SP6 and KI2 points for 3 minutes on the first day of menstruation in the experimental group. The control group did not receive any intervention except for data collection twice a day. The study reported that menstrual discomfort symptoms such as headache and lower back, lower leg, and lower abdomen pain were significantly reduced in the experimental group. Post-test severity scores were lower than pre-test severity scores in the experimental group [41].

A randomized pragmatic trial was performed with women; in the experimental group, participants were reminded by a smartphone application to apply acupressure at the SP6, LI4, and LR3 points by themselves, and the control group received no intervention. The intensity and duration of pain, medication intake, and the number of days with pain were assessed in the first, second, third, and sixth menstruation cycles. Pain intensity, the number of days with pain, and the use of pain medication were lower in the acupressure group in the third and sixth menstruation cycles [15].

\section{Studies of acupressure with devices}

In the USA, a study was conducted with the Relief Brief acupressure device, which is a cotton Lycra panty with lower abdominal and lower back latex foam acupads that provide pressure to acupressure points for 30 minutes. It was found that the device was effective and safe for the treatment of PD. The intensity of menstrual pain, menstrual pain symptoms (abdominal pain, cramps, and backache), and the use of pain medication were decreased in the acupressure group compared with the control group in the first and the second treatment cycle [38]. In another study in the USA, the experimental group received acupressure for 30 minutes with acupuncture-like transcutaneous electrical nerve stimulation (TENS) at the ST36, SP6, BL21, and BL29 points. The intensity of pain was measured before, immediately after, and $30,60,120$, and 180 minutes after acupressure and the next morning upon awakening. There was a statistically significant reduction in pain in the control and experimental groups for all measurements. However, there was no statistically significant difference in pain relief between the groups [37].

\section{Studies of self-acupressure}

In this review, 9 of 28 studies in Iran [5,37], Taiwan $[1,2,22,35,36]$, Germany [15], and China [18] involved selfadministered acupressure, and in the remaining 19 studies, acupressure was applied by trained personnel. In Germany, 1 of 9 studies involved app-based self-acupressure [15]. Acupressure was performed at different points (LI4, SP6, ST36, LR3, and auricular acupressure) and started at different times $[1,2,5,15,18,22,35,36]$. In four studies, pain intensity was reduced in the self-acupressure group compared with the control group, which received no intervention or pressure at the placebo point $[1,5,18,22]$. Furthermore, anxiety associated with dysmenorrhea was reduced following acupressure in one study [37]. In another study, app-based self-acupressure resulted in a decrease in menstrual pain compared with usual care, which was defined as all medical and non-medical treatments [15]. Three of the studies that applied auricular acupressure reported that auricular acupressure relieved menstrual pain and distress in $\operatorname{PD}[2,35,36]$.

\section{DISCUSSION}

This review aimed to determine the effects of acupressure on PD based on experimental studies. The results of 28 studies were categorized under six subheadings (acupressure at the LR3 point, acupressure at the SP6 point, acupressure at auricular points, acupressure at multiple points, acupressure with devices, and self-acupressure). The literature review revealed that acupressure could reduce menstrual symptoms, the severity and duration of menstrual pain, distress, and anxiety in different countries.

In the studies, the total duration of acupressure ranged from 12 and 30 minutes with pressure applied at different points. Acupressure was started at different times before menstruation or after the bleeding day. In addition, pain intensity was measured at different times, i.e., immediately after acupressure, $0.5,1,2,3$, and 4 hours after acupressure, and during several menstrual cycles $(1,2,3,4,6,8$, and 12 months). Moreover, in the experimental studies, various data 
collection tools were used to assess pain (VAS, AMS, MPQ, etc.).

Acupressure can be given by health care providers with appropriate teaching and training. However, various factors could affect outcome measurements. Intervention is given by pressing acupoints with fingers, hands, seeds, or devices using force. However, there is a lack of standard procedure or clinical protocol in acupressure treatment, such as guidelines for the frequency, duration of time, and selection of points. Therefore, further research should be conducted to ensure the consistency of techniques, which would facilitate evidencebased practice [44].

Abaraogu et al. reviewed 6 studies $(n=461)$ that examined SP6 acupressure for the relief of PD symptoms, as well as the patients' experiences with this intervention. According to this meta-analysis, SP6 acupressure performed by trained personnel significantly decreased pain intensity immediately after intervention, and pain relief remained up to 3 hours after intervention. However, self-acupressure required multiple monthly cycles to achieve considerable pain reduction. SP6 acupressure applied by trained personnel appears to be effective for some PD symptoms. The findings indicated that self-administered acupressure is promising for the alleviation of $\mathrm{PD}$ symptoms, and high-quality research is needed [45].

Based on the literature and evidence from clinical trials, practical guidelines for the application of finger pressure and the accuracy of acupoints should be further developed in the future. Health care providers should continue to provide acupressure treatment to relieve pain from PD; however, high-quality trials are needed to develop evidence-based guidelines for acupressure in PD [44]. Furthermore, highquality randomized controlled trials with larger samples are necessary to establish the evidence for acupressure as an effective intervention $[46,47]$.

The study has some limitations. First, the results of quality assessment in the trials were not good, and the efficacy was not estimated. As 14 of the studies had a Jadad score below 2, most of the studies were of low quality. Second, the experimental studies were carried out with different methodological designs, including different acupressure points, outcome measures, and evaluation tools. As a standard protocol was not used, it is difficult to compare the study findings.

\section{CONCLUSIONS}

In this review, 14 of the 28 studies were of low quality according to the Jadad score. However, the methodologies of studies differed in terms of the applied acupressure at different points, times, and intervals, total duration of pressure, sample size, and data collection tools for assessing pain. The evaluated studies in this review demonstrated that anxiety, distress, the severity and duration of menstrual pain, abdominal and back pain, menstrual discomfort, and the severity of systemic symptoms were decreased. In addition, the comfort, relaxation, and satisfaction of women were increased with acupressure at different points. Nevertheless, high-quality research is needed with larger samples.

\section{AUTHORS' CONTRIBUTIONS}

The conception and design of the study: Aslı Karakuş Selçuk, Emre Yanıkkerem. Acquisition of data: Aslı Karakuş Selçuk, Emre Yanıkkerem. Analysis and interpretation of data: Aslı Karakuş Selçuk, Emre Yanıkkerem. Drafting the article or revising it critically for important intellectual content: Aslı Karakuş Selçuk, Emre Yanıkkerem. Final approval of the version to be submitted: Aslı Karakuş Selçuk, Emre Yanıkkerem.

\section{CONFLICT OF INTEREST}

The authors declare no conflict of interest.

\section{ORCID}

Aslı Karakuş Selçuk,

https://orcid.org/0000-0003-1603-4259

Emre Yanikkerem,

https://orcid.org/0000-0001-8909-3597

\section{REFERENCES}

1. Chen HM, Chen CH. Effects of acupressure at the Sanyinjiao point on primary dysmenorrhoea. J Adv Nurs 2004;48:380-7.

2. Yeh ML, Hung YL, Chen HH, Lin JG, Wang YJ. Auricular acupressure combined with an internet-based intervention or alone for primary dysmenorrhea: a control study. Evid Based Complement Alternat Med 2013;2013:316212.

3. Cha NH, Sok SR. Effects of auricular acupressure therapy on primary dysmenorrhea for female high school students in South Korea. J Nurs Scholarsh 2016;48:508-16.

4. Behbahani BM, Ansaripour L, Akbarzadeh M, Zare N, Hadianfard MJ. Comparison of the effects of acupressure and self-care behaviors training on the intensity of primary dysmenorrhea based on McGill pain questionnaire among Shiraz University students. J Res Med Sci 2016;21:104.

5. Bazarganipour F, Taghavi SA, Allan H, Hosseini N, Khosravi A, Asadi R, et al. A randomized controlled clinical trial evaluating quality of life when using a simple acupressure protocol in women with primary dysmenorrhea. Complement Ther Med 2017;34:10-5.

6. Rafique N, Al-Sheikh MH. Prevalence of primary dysmenorrhea 
and its relationship with body mass index. J Obstet Gynaecol Res 2018;44:1773-8.

7. Fernández-Martínez E, Onieva-Zafra MD, Parra-Fernández ML. Lifestyle and prevalence of dysmenorrhea among Spanish female university students. PLoS One 2018;13:e0201894.

8. Chia CF, Lai JH, Cheung PK, Kwong LT, Lau FP, Leung KH, et al. Dysmenorrhoea among Hong Kong university students: prevalence, impact, and management. Hong Kong Med J 2013; 19:222-8.

9. Subasinghe AK, Happo L, Jayasinghe YL, Garland SM, Gorelik A, Wark JD. Prevalence and severity of dysmenorrhoea, and management options reported by young Australian women. Aust Fam Physician 2016;45:829-34.

10. Aktaş D. Prevalence and factors affecting dysmenorrhea in female university students: effect on general comfort level. Pain Manag Nurs 2015;16:534-43.

11. Midilli TS, Yasar E, Baysal E. Dysmenorrhea characteristics of female students of health school and affecting factors and their knowledge and use of complementary and alternative medicine methods. Holist Nurs Pract 2015;29:194-204.

12. Potur DC, Bilgin NC, Komurcu N. Prevalence of dysmenorrhea in university students in Turkey: effect on daily activities and evaluation of different pain management methods. Pain Manag Nurs 2014;15:768-77.

13. Yilmaz B, Sahin N. The effects of a dysmenorrhea support program on university students who had primary dysmenorrhea: a randomized controlled study. J Pediatr Adolesc Gynecol 2020;33:285-90.

14. Kafaei Atrian M, Abbaszadeh F, Sarvieh M, Sarafraz N, Asghari Jafarabadi M. Investigatingthe effect of pressure on third liver point on primary dysmenorrhea: a randomized controlled clinical trial. Iran Red Crescent Med J 2013;15:848-53.

15. Blödt S, Pach D, Eisenhart-Rothe SV, Lotz F, Roll S, Icke K, et al. Effectiveness of app-based self-acupressure for women with menstrual pain compared to usual care: a randomized pragmatic trial. Am J Obstet Gynecol 2018;218:227.e1-9.

16. Jun EM, Chang S, Kang DH, Kim S. Effects of acupressure on dysmenorrhea and skin temperature changes in college students: a non-randomized controlled trial. Int J Nurs Stud 2007;44:973-81.

17. Kashefi F, Ziyadlou S, Khajehei M, Ashraf AR, Fadaee AR, Jafari P. Effect of acupressure at the Sanyinjiao point on primary dysmenorrhea: a randomized controlled trial. Complement Ther Clin Pract 2010;16:198-202.

18. Wong CL, Lai KY, Tse HM. Effects of SP6 acupressure on pain and menstrual distress in young women with dysmenorrhea. Complement Ther Clin Pract 2010;16:64-9.

19. Charandabi SM, Nashtaei MS, Kamali S, Majlesi R. The effect of acupressure at the Sanyinjiao point (SP6) on primary dysmenorrhea in students resident in dormitories of Tabriz. Iran J Nurs Midwifery Res 2011;16:309-17.
20. Mirbagher-Ajorpaz N, Adib-Hajbaghery M, Mosaebi F. The effects of acupressure on primary dysmenorrhea: a randomized controlled trial. Complement Ther Clin Pract 2011;17:33-6.

21. Tanriverdi S, Saritaş S. The effect of acupressure on postoperative pain of lumbar disc hernia: a quasi-experimental study. Complement Ther Clin Pract 2018;32:12-6.

22. Chen HM, Chen CH. Effects of acupressure on menstrual distress in adolescent girls: a comparison between Hegu-Sanyinjiao matched points and Hegu, Zusanli single point. J Clin Nurs 2010;19:998-1007.

23. Wang MC, Hsu MC, Chien LW, Kao CH, Liu CF. Effects of auricular acupressure on menstrual symptoms and nitric oxide for women with primary dysmenorrhea. J Altern Complement Med 2009;15:235-42.

24. Gharloghi S, Torkzahrani S, Akbarzadeh AR, Heshmat R. The effects of acupressure on severity of primary dysmenorrhea. Patient Prefer Adherence 2012;6:137-42.

25. Chen HM, Wang HH, Chiu MH, Hu HM. Effects of acupressure on menstrual distress and low back pain in dysmenorrheic young adult women: an experimental study. Pain Manag Nurs 2015;16:188-97.

26. Bazarganipour F, Lamyian M, Heshmat R, Abadi MA, Taghavi A. A randomized clinical trial of the efficacy of applying a simple acupressure protocol to the Taichong point in relieving dysmenorrhea. Int J Gynaecol Obstet 2010;111:105-9.

27. Liberati A, Altman DG, Tetzlaff J, Mulrow C, Gøtzsche PC, Ioannidis JP, et al. The PRISMA statement for reporting systematic reviews and meta-analyses of studies that evaluate health care interventions: explanation and elaboration. PLoS Med 2009;6:e1000100.

28. Chung YC, Chen HH, Yeh ML. Acupoint stimulation intervention for people with primary dysmenorrhea: systematic review and meta-analysis of randomized trials. Complement Ther Med 2012;20:353-63.

29. Olivo SA, Macedo LG, Gadotti IC, Fuentes J, Stanton T, Magee DJ. Scales to assess the quality of randomized controlled trials: a systematic review. Phys Ther 2008;88:156-75.

30. Jadad AR, Moore RA, Carroll D, Jenkinson C, Reynolds DJ, Gavaghan DJ, et al. Assessing the quality of reports of randomized clinical trials: is blinding necessary? Control Clin Trials 1996;17:1-12.

31. Halpern SH, Douglas MJ. Appendix: Jadad scale for reporting randomized controlled trials. In: Halpern SH, Douglas MJ, eds. Evidence-based Obstetric Anesthesia. Oxford: Blackwell Publishing Ltd, 2005:237-8.

32. Pouresmail Z, Ibrahimzadeh R. Effects of acupressure and ibuprofen on the severity of primary dysmenorrhea. J Tradit Chin Med 2002;22:205-10.

33. Zafari M, Tofighi M, Aghamohammady A, Behmanesh F, Rakhshaee Z. Comparison of the effect of acupressure, fish oil capsules and ibuprofen on treatment of primary dysmenorrheal. 
Afr J Pharm Pharmacol 2011;5:1115-9.

34. Kafaei-Atrian M, Mirbagher-Ajorpaz N, Sarvieh M, Sadat Z, Asghari-Jafarabadi M, Solhi M. The effect of acupressure at third liver point on the anxiety level in patients with primary dysmenorrhea. Iran J Nurs Midwifery Res 2016;21:142-6.

35. Wang YJ, Hsu CC, Yeh ML, Lin JG. Auricular acupressure to improve menstrual pain and menstrual distress and heart rate variability for primary dysmenorrhea in youth with stress. Evid Based Complement Alternat Med 2013;2013:138537.

36. Yeh ML, Hung YL, Chen HH, Wang YJ. Auricular acupressure for pain relief in adolescents with dysmenorrhea: a placebocontrolled study. J Altern Complement Med 2013;19:313-8.

37. Lewers D, Clelland JA, Jackson JR, Varner RE, Bergman J. Transcutaneous electrical nerve stimulation in the relief of primary dysmenorrhea. Phys Ther 1989;69:3-9.

38. Taylor D, Miaskowski C, Kohn J. A randomized clinical trial of the effectiveness of an acupressure device (relief brief) for managing symptoms of dysmenorrhea. J Altern Complement Med 2002;8:357-70.

39. Archana R, Philominal V, Thirumeni S. Effect of acupressure and changes in heart rate variability in dysmenorrhoea. Recent Res Sci Technol 2011;3(10):1-6.

40. Elakkiya C. An experimental study to assess the effectiveness of acupressure on dysmenorrhea among adolescent girls in Hilton Matriculation Higher Secondary School at Chrompet, Chennai.
Int J Innov Res Dev 2015;4:8-10.

41. Soja SL, Nayak BS, Lobo DJ. A study to evaluate the effectiveness of acupressure on menstrual discomforts among adolescent girls. Int J Nurs Educ 2011;3:107-10.

42. Maryanti SA, Keb M. The effectiveness of acupressure in reducing the pain intensity of dysmenorrhea. IOSR J Nurs Health Sci 2017;6:77-83.

43. WHO Regional Office for the Western Pacific. Standard acupuncture nomenclature, part 1. Rev. ed.. Available at: https:// apps.who.int/iris/bitstream/handle/10665/207635/Standard_ acupuncture_nomenclature_1991_partI_eng.pdf?sequence $=1 \&$ isAllowed $=y$ [Date accessed: June 24, 2020]

44. Chen YW, Wang HH. The effectiveness of acupressure on relieving pain: a systematic review. Pain Manag Nurs 2014;15: 539-50.

45. Abaraogu UO, Igwe SE, Tabansi-Ochiogu CS. Effectiveness of SP6 (Sanyinjiao) acupressure for relief of primary dysmenorrhea symptoms: a systematic review with meta- and sensitivity analyses. Complement Ther Clin Pract 2016;25:92-105.

46. Cho SH, Hwang EW. Acupressure for primary dysmenorrhoea: a systematic review. Complement Ther Med 2010;18:49-56.

47. Song HJ, Seo HJ, Lee H, Son H, Choi SM, Lee S. Effect of selfacupressure for symptom management: a systematic review. Complement Ther Med 2015;23:68-78. 